\title{
MONOTONICALLY CONVERGENT OPTIMAL CONTROL THEORY OF QUANTUM SYSTEMS UNDER A NONLINEAR INTERACTION WITH THE CONTROL FIELD
}

\author{
M. LAPERT ${ }^{1}$, R. TEHINI ${ }^{1}$, G. TURINICI ${ }^{2}$, D. SUGNY ${ }^{1}$
}

\begin{abstract}
We consider the optimal control of quantum systems interacting non-linearly with an electromagnetic field. We propose new monotonically convergent algorithms to solve the optimal equations. The monotonic behavior of the algorithm is ensured by a non-standard choice of the cost which is not quadratic in the field. These algorithms can be constructed for pure and mixedstate quantum systems. The efficiency of the method is shown numerically on molecular orientation with a nonlinearity of order 3 in the field. Discretizing the amplitude and the phase of the Fourier transform of the optimal field, we show that the optimal solution can be well-approximated by pulses that could be implemented experimentally.
\end{abstract}

\section{INTRODUCTION}

The control of quantum dynamics induced by an intense laser field continue to be a challenge to both experiment and theory [1, 2, 3. In this context, Optimal Control Theory (OCT) is an efficient tool for designing laser pulses able to control quantum processes $[4,5,6,7,8,9,10,11,12,13,14$. By construction, the optimal field is the field steering a dynamical system from the initial state to a desired target state and minimizing a cost functional which generally penalizes the energy or the duration of the field. Different methods have been developed to solve the optimal equations [11, 15]. Among others, monotonically convergent iterative schemes proposed by Tannor et al. [16] and Rabitz et al. [4, 17, 18, have been applied with success to a variety of physical and chemical processes $4,19,20,21,22$. These algorithms have the particularity to guarantee the increase of the cost functional at each step of iteration. In this paper, we will consider the Rabitz formulation of iterative algorithms [17. First introduced to treat pure-state quantum systems, these schemes have been extended and applied to mixed-state quantum systems, dissipative ones [7, 8, and non-markovian dynamics [23. A majority of works has considered a linear interaction between the quantum system and the electromagnetic field. This linear interaction corresponds, for molecular systems, to the first order dipolar approximation (permanent dipole moment). Due to the intensity of the field or to the particular structure of the problem, some systems need to go beyond this approximation [24, 25, 26, 27. A typical example is given by the control of molecular orientation and alignment of a linear molecule by non-resonant laser pulses [28, 29, 24, 27]. When averaging over the rapid oscillations of the field, one observes that the permanent dipole moment plays no role in the control of the dynamics. In this case, molecular alignment and orientation are obtained

Date: October 31, 2018. 
via the polarizability and the hyperpolarizability terms of the interaction Hamiltonian (see [30] for information on the controllability of these systems). From a methodological point of view, the natural question arises of whether one can apply monotonically convergent algorithms to such systems interacting non-linearly with the field.

The goal of this work is to answer this question by proposing new monotonic algorithms when an arbitrary nonlinearity is considered. A key ingredient to ensure the monotonic convergence of the algorithms is to consider a non-standard cost functional which instead of penalizing the intensity of the field, i.e., the square of the electric field penalizes a higher exponent which depends on the order of the non-linearity. Note that a similar question has been treated in [12. A family of algorithms different from those proposed in this paper has been developed. In algorithms of [12, the cost is quadratic in the field and the control is decomposed into $n$ components for a nonlinearity of order $n$. Thus, for each iteration of the algorithm, $2 n$ numerical resolutions of the time-dependent Schrödinger equation are required: $n$ for the wave function and $n$ for the Lagrange multiplier. On the contrary, in this work, we use only one component for the control field but at the price of modifying the cost functional. We construct monotonically convergent algorithms for pure and mixed-state quantum systems but they can be generalized straightforwardly to dissipative dynamics. We test the efficiency of these algorithms on the orientation dynamics of a linear molecule with non-linearity of order 3 corresponding to the hyperpolarizability terms of the molecule [27. We use as target states the states which maximize the orientation in a finite-dimensional restriction of the Hilbert space. Several works have pointed out the role of these target states which both optimize the field-free orientation and its duration [31, 32, 33, 34. Promising results have been obtained both for pure and mixed-state quantum systems corresponding to zero and non-zero temperatures.

Finally, we also analyze the structure of the Fourier transform of optimal control pulses. Our aim is to show that the optimal solutions can be well-approximated by pulses that could be implemented experimentally [35, 36, 37, 38. Such pulses, tailored by genetic algorithms, have been successfully applied for experimentally and theoretically controlling different molecular processes [36, 37, 38, 39, 40, 41. In the frequency domain, they are characterized by the fact that both the amplitude and the phase of the Fourier transform (but only for a finite number of frequencies equally distributed over a given frequency interval) are optimized [39, 40, 41]. This choice corresponds to the types of pulses that can be implemented by liquid crystal pulse shapers. As an alternative, we use in this paper the results of our monotonic optimization algorithms to construct such pulses. Note that we do not adopt filtering techniques in the iterative algorithm, which have been proposed by several works (see 11] and references therein). The idea consists generally in applying a filter to the control field at each iteration in order to satisfy spectral constraints. This filtering has the drawback that it does not generally yield a monotonic convergence of the algorithm. Instead, we propose to use a simpler solution. Starting from the optimal solution obtained by the monotonic algorithm, we discretize the phase and the amplitude of its Fourier transform into 640 points or less (640 points correspond to the number of pixels usually used in pulse-shaping experiments). From this discretization, we then construct a piecewise constant Fourier transform and a new time-dependent electric field by an inverse Fourier transform [40, 41]. 
We finally compare the optimal result and the one obtained with the discretized field. We show that the difference between the two results is negligible when the structure of the optimal field is sufficiently simple or equivalently when the number of pixels is sufficiently large.

This paper is organized as follows. We first present the model system in Sec. 2.1. We determine in Sec. 2.2 the polynomial equation that must be satisfied by the optimal field. We then outline in Sec. 2.3 the principle of monotonically convergent algorithms for nonlinear interaction both in pure and mixed-state cases. A special attention is paid to the different choices and to the flexibility of the method. Generalizing the proofs of Refs. [17] and [22, we show the monotonic behavior of the algorithms. Section 3 is devoted to the application of these strategies to molecular orientation. The results are presented at $T=0 \mathrm{~K}$ (Sec. 3.2) and $T \neq 0 \mathrm{~K}$ (Sec. 3.5) for the standard case (i.e. with a linear interaction term) and at $T=0 \mathrm{~K}$ for the averaging case (Sec. 3.3). We also propose an algorithm well-suited to the simultaneous optimization of two laser fields. An example is given by the non-resonant control of molecular orientation by two-color laser pulses [27]. We finally examine in Sec. 3.4 the structure of the Fourier transform of the optimal fields.

\section{Optimal Control Theory}

The goal of this section is to propose monotonically convergent algorithms suited to quantum systems interacting nonlinearly with the control field. To simplify the discussion, we consider the case of pure-state quantum systems. Following Ref. [8] and the formalism of super-operator, the proof can be straightforwardly extended to mixed-state quantum systems (see for that purpose Sec. 3.5). Optimal control theory is invoked in order to maximize the projection onto a target state, but it could be equivalently defined for maximizing the expectation value of a given observable. The proof is a generalization of the standard proof for linear interaction [17] and of the proof given in Ref. [22].

2.1. The model system. We consider a quantum system interacting with an electromagnetic field whose dynamics is governed by the following time-dependent Schrödinger equation

$$
i \frac{\partial}{\partial t}|\psi(t)\rangle=\hat{H}(t)|\psi(t)\rangle
$$

which is written in units such that $\hbar=1$. The Hamiltonian $\hat{H}(t)$ of the system is given by

$$
\hat{H}(t)=\hat{H}_{0}-\hat{\mu} E(t)-\hat{\alpha} E(t)^{2}-\hat{\beta} E(t)^{3} \cdots,
$$

where $\hat{H}_{0}$ is the field-free Hamiltonian. The other terms describe the interaction between the system and the laser field $E(t)$. This interaction is written as a polynomial expansion in $E(t)$ whose coefficients are the operators $\hat{\mu}, \hat{\alpha}, \hat{\beta} \cdots$. For a linear molecule interacting with a linearly polarized laser field, the different operators $\hat{\mu}, \hat{\alpha}$ and $\hat{\beta}$ are associated to the permanent dipole moment $\mu_{0}$, the polarizability components $\alpha_{\|}$and $\alpha_{\perp}$ and the hyperpolarizability components $\beta_{\|}$and $\beta_{\perp}$ of the molecule 27. These different molecular constants will be used in numerical computations of Sec. 3 . 
2.2. Critical point. Let $\left|\phi_{0}\right\rangle$ and $\left|\phi_{f}\right\rangle$ be the initial and the target states of the control. We denote by $t_{f}$ the duration of the control. We define the optimal control theory through the following cost functional:

$$
J=\left|\left\langle\phi_{f} \mid \psi\left(t_{f}\right)\right\rangle\right|^{2}-\int_{0}^{t_{f}} \lambda E(t)^{2 n} d t,
$$

where $n$ is a positive integer. The even exponent of the integrand and the choice $\lambda \geq 0$ ensure the negativity of the second term of Eq. (3). $n$ is taken equal to 1 for a linear interaction but we will see that, in order to obtain monotonic algorithms, larger values of $n$ have to be considered when the system interacts non-linearly with the field. $\lambda$ is a penalty factor which weights the importance of the laser fluence. Following [42, we will replace in Sec. 3 this constant by $\lambda / s(t)$, where $s(t)=\sin ^{2}\left(\pi t / t_{f}\right)$, which penalizes more strongly the amplitude of the pulse at the beginning and at the end of the control. This allows to obtain more realistic optimal solutions.

We introduce the augmented cost functional $\bar{J}$ which is defined through the adjoint state $|\chi(t)\rangle$ as follows

$$
\bar{J} \neq 4\rangle\left.\left\langle\phi_{f} \mid \psi\left(t_{f}\right)\right\rangle\right|^{2}-\int_{0}^{t_{f}} \lambda E(t)^{2 n} d t-2 \Im\left[\left\langle\psi\left(t_{f}\right) \mid \phi_{f}\right\rangle \int_{0}^{t_{f}}\left\langle\chi(t)\left|\left(i \frac{\partial}{\partial t}-\hat{H}\right)\right| \psi(t)\right\rangle d t\right],
$$

where $\Im$ denotes the imaginary part. The optimal electric field is solution of the equation

$$
\frac{\delta \bar{J}}{\delta E(t)}=0
$$

which is a polynomial equation in $E(t)$ :

(6) $2 n \lambda E(t)^{2 n-1}+2 \Im\left[\left\langle\psi\left(t_{f}\right) \mid \phi_{f}\right\rangle\left\langle\chi(t)\left|\hat{\mu}+2 \hat{\alpha} E(t)+3 \hat{\beta} E(t)^{2}\right| \psi(t)\right\rangle\right]=0$.

The second term of Eq. (6) can be modified by using the fact that

$$
\frac{d}{d t}\langle\psi(t) \mid \chi(t)\rangle=0
$$

The equation for the optimal field finally reads

(8) $2 n \lambda E(t)^{2 n-1}+2 \Im\left[\langle\psi(t) \mid \chi(t)\rangle\left\langle\chi(t)\left|\hat{\mu}+2 \hat{\alpha} E(t)+3 \hat{\beta} E(t)^{2}\right| \psi(t)\right\rangle\right]=0$.

Setting the variations of $\bar{J}$ with respect to $|\psi(t)\rangle$ and $|\chi(t)\rangle$ to 0 ensures that $|\psi(t)\rangle$ and $|\chi(t)\rangle$ satisfy the Schrödinger equation (11). To summarize, an extremum of $\bar{J}$ satisfies the equations

$$
\begin{aligned}
& \left(i \frac{\partial}{\partial t}-\hat{H}(t)\right)|\psi(t)\rangle=0 \\
& |\psi(0)\rangle=\left|\phi_{0}\right\rangle
\end{aligned}
$$

for the state $|\psi(t)\rangle$ and

$$
\begin{aligned}
& \left(i \frac{\partial}{\partial t}-\hat{H}(t)\right)|\chi(t)\rangle=0 \\
& \left|\chi\left(t_{f}\right)\right\rangle=\left|\phi_{f}\right\rangle
\end{aligned}
$$

for the adjoint state $|\chi(t)\rangle$, the control field $E(t)$ being solution of Eq. (8). 
2.3. Monotonically convergent algorithm. We describe different iterative algorithms to solve the optimal equations of Sec. 2.2. To simplify the presentation of computations, we consider nonlinearity of order 3 and a cost which is quartic in the field $(n=2)$.

At step $k \geq 1$ of the algorithm, the system is described by the quadruplet $\left(\left|\psi_{k}(t)\right\rangle,\left|\chi_{k-1}(t)\right\rangle, E_{k}(t), \tilde{E}_{k-1}(t)\right)$ where $\left|\psi_{k}(t)\right\rangle$ is the state of the system, $\left|\chi_{k-1}(t)\right\rangle$ the adjoint state, $E_{k}(t)$ and $\tilde{E}_{k-1}(t)$ the electric fields associated respectively to the forward propagation of $\left|\psi_{k}(t)\right\rangle$ and to the backward propagation of $\left|\chi_{k-1}(t)\right\rangle$. $\left|\psi_{k}(t)\right\rangle$ and $\left|\chi_{k-1}(t)\right\rangle$ are solutions of the following time-dependent Schrödinger equations

$$
i \frac{\partial}{\partial t}\left|\psi_{k}(t)\right\rangle=\hat{H}\left(E_{k}\right)\left|\psi_{k}(t)\right\rangle
$$

and

$$
i \frac{\partial}{\partial t}\left|\chi_{k-1}(t)\right\rangle=\hat{H}\left(\tilde{E}_{k-1}\right)\left|\chi_{k-1}(t)\right\rangle
$$

where $\hat{H}(E(t))=\hat{H}_{0}-\hat{\mu} E(t)-\hat{\alpha} E(t)^{2}-\hat{\beta} E(t)^{3}$. For $\left|\psi_{k}(t)\right\rangle$, we impose the initial condition $\left|\psi_{k}(0)\right\rangle=\left|\phi_{0}\right\rangle$ and for $\left|\chi_{k-1}(t)\right\rangle$ the final condition $\left|\chi_{k-1}\left(t_{f}\right)\right\rangle=\left|\phi_{f}\right\rangle$. The iteration is initiated by a trial electric field $E_{0}(t)$. At step 0 of the algorithm, we propagate forward the state $\left|\psi_{0}(t)\right\rangle$ with the electric field $E_{0}(t)$. The cost functional $J_{k}$ at step $k$ is defined by

$$
J_{k}=\left|\left\langle\phi_{f} \mid \psi_{k}\left(t_{f}\right)\right\rangle\right|^{2}-\int_{0}^{t_{f}} \lambda E_{k}^{4} .
$$

The algorithm determines the quadruplet $\left(\left|\psi_{k+1}(t)\right\rangle,\left|\chi_{k}(t)\right\rangle, E_{k+1}(t), \tilde{E}_{k}(t)\right)$ at step $k+1$ from the one at step $k$. This is done by requiring that the variation $\Delta J=J_{k+1}-J_{k}$ of the cost $J$ from step $k$ to step $k+1$ is positive and that the limits (if they exist) of the sequences $\left(E_{k}\right)_{k \in \mathbb{N}}$ and $\left(\tilde{E}_{k}\right)_{k \in \mathbb{N}}$ are solutions of Eq. (8).

For that purpose, we introduce the functions $P_{k+1}(t)=\left|\left\langle\chi_{k}(t) \mid \psi_{k+1}(t)\right\rangle\right|^{2}$ and $\tilde{P}_{k+1}(t)=\left|\left\langle\chi_{k+1}(t) \mid \psi_{k+1}(t)\right\rangle\right|^{2}$. Differentiating with respect to time these two functions leads to

$$
\left(\frac{d}{d t}\right) P_{k+1}(t)=\mu_{k, k+1}\left(\tilde{E}_{k}-E_{k+1}\right)+\alpha_{k, k+1}\left(\tilde{E}_{k}^{2}-E_{k+1}^{2}\right)+\beta_{k, k+1}\left(\tilde{E}_{k}^{3}-E_{k+1}^{3}\right)
$$

for $P_{k+1}$ and to

$$
\begin{aligned}
& \frac{d}{d t} \tilde{P}_{k+1}(t)=\mu_{k+1, k+1}\left(\tilde{E}_{k+1}-E_{k+1}\right)+\alpha_{k+1, k+1}\left(\tilde{E}_{k+1}^{2}-E_{k+1}^{2}\right) \\
& +\beta_{k+1, k+1}\left(\tilde{E}_{k+1}^{3}-E_{k+1}^{3}\right)
\end{aligned}
$$

for $\tilde{P}_{k+1}$. In Eqs. (14) and (15), we have introduced the notation

$$
A_{k, k^{\prime}}=2 \Im\left[\left\langle\psi_{k^{\prime}}(t) \mid \chi_{k}(t)\right\rangle\left\langle\chi_{k}(t)|\hat{A}| \psi_{k^{\prime}}(t)\right\rangle\right]
$$

for a given observable $\hat{A}$. The functions $P_{k}$ and $\tilde{P}_{k}$ fulfill by definition the following relations:

$$
\begin{aligned}
& \tilde{P}_{k+1}\left(t_{f}\right)=\left|\left\langle\phi_{f} \mid \psi_{k+1}\left(t_{f}\right)\right\rangle\right|^{2}=P_{k+1}\left(t_{f}\right) \\
& \tilde{P}_{k+1}(0)=\left|\left\langle\chi_{k+1}(0) \mid \phi_{0}\right\rangle\right|^{2}=P_{k+2}(0),
\end{aligned}
$$


and a direct integration gives

$$
P_{k}\left(t_{f}\right)=P_{k}(0)+\int_{0}^{t_{f}} \frac{d P_{k}(t)}{d t} d t .
$$

The variation $\Delta J$ is given by

$\Delta J \neq 1 g_{\eta \neq+1}-J_{k}=\left|\left\langle\phi_{f} \mid \psi_{k+1}\left(t_{f}\right)\right\rangle\right|^{2}-\left|\left\langle\phi_{f} \mid \psi_{k}\left(t_{f}\right)\right\rangle\right|^{2}-\int_{0}^{t_{f}} \lambda\left[E_{k+1}(t)^{4}-E_{k}(t)^{4}\right] d t$.

Using the fact that $\left|\left\langle\phi_{f} \mid \psi_{k+1}\left(t_{f}\right)\right\rangle\right|^{2}-\left|\left\langle\phi_{f} \mid \psi_{k}\left(t_{f}\right)\right\rangle\right|^{2}=P_{k+1}\left(t_{f}\right)-P_{k}\left(t_{f}\right)$ and Eqs. (17), one deduces that $\Delta J=P_{1}+P_{2}$ where

$$
\begin{aligned}
& P_{1}=-\int_{0}^{t_{f}} \lambda\left[E_{k+1}^{4}-\tilde{E}_{k}^{4}\right]+ \\
& \int_{0}^{t_{f}}\left[\left(\tilde{E}_{k}-E_{k+1}\right) \mu_{k, k+1}+\left(\tilde{E}_{k}^{2}-E_{k+1}^{2}\right) \alpha_{k, k+1}+\left(\tilde{E}_{k}^{3}-E_{k+1}^{3}\right) \beta_{k, k+1}\right] d t
\end{aligned}
$$

and

$$
\begin{aligned}
& P_{2}=\int_{0}^{t_{f}} \lambda\left[E_{k}^{4}-\tilde{E}_{k}^{4}\right]- \\
& \int_{0}^{t_{f}}\left[\left(\tilde{E}_{k}-E_{k}\right) \mu_{k, k}+\left(\tilde{E}_{k}^{2}-E_{k}^{2}\right) \alpha_{k, k}+\left(\tilde{E}_{k}^{3}-E_{k}^{3}\right) \beta_{k, k}\right] d t .
\end{aligned}
$$

To ensure the monotonic behavior of the algorithm, we choose the fields $E_{k+1}$ and $\tilde{E}_{k}$ such that the integrals $P_{1}$ and $P_{2}$ are positive. A sufficient condition is to impose that the two integrands $\mathcal{P}_{1}$ and $\mathcal{P}_{2}$ associated to $P_{1}$ and $P_{2}$ are positive [22]. To be more precise, we first determine $\tilde{E}_{k}$ from $E_{k}$ such that $P_{2}$ is positive and then we determine $E_{k+1}$ from $\tilde{E}_{k}$ such that $P_{1}$ is positive. $\left|\psi_{k+1}\right\rangle$ and $\left|\chi_{k}\right\rangle$ are computed from a forward and a backward propagation with the fields $E_{k+1}$ and $\tilde{E}_{k}$.

Starting from these conditions, we introduce two algorithms.

\section{Algorithm I:}

$\mathcal{P}_{1}$ and $\mathcal{P}_{2}$ are respectively viewed as functions of $E_{k+1}$ and $\tilde{E}_{k} . \quad E_{k+1}$ and $\tilde{E}_{k}$ are defined as the control fields which maximize $\mathcal{P}_{1}$ and $\mathcal{P}_{2}$. The maxima of these polynomials are positive since $\mathcal{P}_{1}\left(\tilde{E}_{k}\right)=0$ and $\mathcal{P}_{2}\left(E_{k}\right)=0$. As already mentioned, we first determine for each time $t$ the maximum of $\mathcal{P}_{2}$ and then the one of $\mathcal{P}_{1}$. The integer $n$ of the cost is chosen sufficiently large to ensure that the fields which maximize $\mathcal{P}_{1}$ and $\mathcal{P}_{2}$ are finite. This means that we choose $n$ such that the terms $-\lambda E_{k+1}^{2 n}$ and $-\lambda \tilde{E}_{k}^{2 n}$ are the monomials of higher degree in $\mathcal{P}_{1}$ and $\mathcal{P}_{2}$. We then have:

$$
\begin{aligned}
& \lim _{E_{k+1} \rightarrow \pm \infty} \mathcal{P}_{1}\left(E_{k+1}\right)=0 \\
& \lim _{\tilde{E}_{k} \rightarrow \pm \infty} \mathcal{P}_{2}\left(\tilde{E}_{k}\right)=0
\end{aligned}
$$

which satisfies the requirement. For nonlinearity of order 3 , the choice $n=2$ is sufficient. Finally, if we assume that the algorithm converges then we can check that the limits of the sequences $\left(E_{k}\right)_{k \in \mathbb{N}}$ and $\left(\tilde{E}_{k}\right)_{k \in \mathbb{N}}$ are solutions of Eq. (8). For that, we respectively differentiate $\mathcal{P}_{1}$ and $\mathcal{P}_{2}$ with respect to $E_{k+1}$ and $\tilde{E}_{k}$. We next replace in the derivatives of $\mathcal{P}_{1}$ and $\mathcal{P}_{2}, E_{k+1}, E_{k}$ and $\tilde{E}_{k}$ by $E,\left|\psi_{k+1}\right\rangle,\left|\psi_{k}\right\rangle$ by $|\psi\rangle$ and $\left|\chi_{k}\right\rangle$ by $|\chi\rangle$. It is then straightforward to see that the limit $E(t)$ satisfies the optimal equation (8). 


\section{Algorithm II:}

We first write $\mathcal{P}_{1}$ and $\mathcal{P}_{2}$ as follows:

$$
\begin{aligned}
& \mathcal{P}_{1}=\left(E_{k+1}-\tilde{E}_{k}\right)\left[-\lambda\left(E_{k+1}^{3}+E_{k+1}^{2} \tilde{E}_{k}+E_{k+1} \tilde{E}_{k}^{2}+\tilde{E}_{k}^{3}\right)\right. \\
& \left.-\mu_{k, k+1}-\alpha_{k, k+1}\left(E_{k+1}+\tilde{E}_{k}\right)-\beta_{k, k+1}\left(E_{k+1}^{2}+\tilde{E}_{k} E_{k+1}+\tilde{E}_{k}^{2}\right)\right],
\end{aligned}
$$

and

$$
\begin{aligned}
& \mathcal{P}_{2}=\left(\tilde{E}_{k}-E_{k}\right)\left[-\lambda\left(\tilde{E}_{k}^{3}+\tilde{E}_{k}^{2} E_{k}+E_{k}^{2} \tilde{E}_{k}+E_{k}^{3}\right)\right. \\
& \left.-\mu_{k, k}-\alpha_{k, k}\left(\tilde{E}_{k}-E_{k}\right)-\beta_{k, k}\left(\tilde{E}_{k}^{2}+E_{k} \tilde{E}_{k}+E_{k}^{2}\right)\right] .
\end{aligned}
$$

We then introduce two positive constants $\eta_{1}$ and $\eta_{2}$ by setting:

$$
\begin{aligned}
& E_{k+1}-\tilde{E}_{k}=\eta_{1}\left[-\lambda\left(E_{k+1}^{3}+E_{k+1}^{2} \tilde{E}_{k}+E_{k+1} \tilde{E}_{k}^{2}+\tilde{E}_{k}^{3}\right)\right. \\
& \left.-\mu_{k, k+1}-\alpha_{k, k+1}\left(E_{k+1}+\tilde{E}_{k}\right)-\beta_{k, k+1}\left(E_{k+1}^{2}+\tilde{E}_{k} E_{k+1}+\tilde{E}_{k}^{2}\right)\right],
\end{aligned}
$$

and

$$
\begin{aligned}
& \tilde{E}_{k}-E_{k}=\eta_{2}\left[-\lambda\left(\tilde{E}_{k}^{3}+\tilde{E}_{k}^{2} E_{k}+E_{k}^{2} \tilde{E}_{k}+E_{k}^{3}\right)\right. \\
& \left.-\mu_{k, k}-\alpha_{k, k}\left(\tilde{E}_{k}+E_{k}\right)-\beta_{k, k}\left(\tilde{E}_{k}^{2}+E_{k} \tilde{E}_{k}+E_{k}^{2}\right)\right] .
\end{aligned}
$$

Equations. (24) and (25) are viewed respectively as equations in $E_{k+1}$ and $\tilde{E}_{k} . E_{k+1}$ and $\tilde{E}_{k}$ are defined as one of the solutions of these two equations. By definition of the constants $\eta_{1}$ and $\eta_{2}$, the values of $\mathcal{P}_{1}$ and $\mathcal{P}_{2}$ for these fields are positive. The integer $n$ is chosen sufficiently large to ensure that Eqs. (24) and (25) always have a real solution respectively in $E_{k+1}$ and $\tilde{E}_{k}$. For nonlinearity of order 3 , it is sufficient to take $n=2$. When Eqs. (24) and (25) have more than one real solution at time $t$, we numerically choose the solution that is closest to the one at time $t-d t$ (for the forward propagation) or $t+d t$ (for the backward propagation). The processus is initiated by imposing that $E_{k}(0)=0$ and $\tilde{E}_{k}\left(t_{f}\right)=0$. This allows one to obtain smooth optimal fields without discontinuity. As for the algorithm I, we can check that the limits of the sequences $\left(E_{k}\right)_{k \in \mathbb{N}}$ and $\left(\tilde{E}_{k}\right)_{k \in \mathbb{N}}$ satisfy Eq. (8). This can be done by replacing $\tilde{E}_{k}$ and $E_{k+1}$ by $E$ in Eqs. (24) and (25).

In the two cases, the structure of the algorithms can be summarized as follows. At step $k+1$, we propagate backward in time the adjoint state $\left|\chi_{k}\right\rangle$ with the field $\tilde{E}_{k}$ determined from $P_{2}$. We then compute the forward evolution of $\left|\psi_{k+1}\right\rangle$ from $\left|\phi_{0}\right\rangle$. For this second propagation, we use the field $E_{k+1}$ defined from $P_{1}$. Note that a simpler solution which gives a slower convergence consists in choosing $\tilde{E}_{k}=E_{k}$, i.e., to propagate $\left|\psi_{k}\right\rangle$ and $\left|\chi_{k}\right\rangle$ with the same field.

\section{Control of molecular orientation}

3.1. Introduction. In this section, we investigate the control of orientation dynamics of a diatomic molecule driven by an electromagnetic field [28, 29]. This control is taken as a prototype to test the efficiency of the algorithm. The application of OCT to molecular alignment and orientation is relatively recent [22, 12, 43]. One of the main results of Ref. 22] is that the optimal oriented state (see below for a definition) is reached by rotational ladder climbing, i.e., by successive rotational excitations. The corresponding optimal pulse is however very long, of the order of 20 rotational periods, which could be problematic for practical applications. We consider shorter durations in this paper of the order of the rotational period $T_{p e r}$. We have chosen $t_{f}=T_{p e r}$ but other durations can be considered. Note that for 
controls much shorter than $T_{p e r}$, the optimal solution is very close to the kick mechanism largely explored using the sudden-impact model [44]. The $C O$ molecule is taken as an example. The units used are atomic units unless otherwise specified.

The molecule is described in a rigid-rotor approximation interacting with a linearly polarized laser pulse nonresonant with vibronic frequencies. In this case, the Hamiltonian $\hat{H}$ can be written as follows [4, 27.

$$
\begin{aligned}
& \hat{H}=B \hat{J}^{2}-\mu_{0} E(t) \cos \theta-\frac{1}{2}\left[\left(\alpha_{\|}-\alpha_{\perp}\right) \cos ^{2} \theta+\alpha_{\perp}\right] E(t)^{2} \\
& -\frac{1}{6}\left[\left(\beta_{\|}-3 \beta_{\perp}\right) \cos ^{3} \theta+3 \beta_{\perp} \cos \theta\right] E(t)^{3},
\end{aligned}
$$

where $B$ and $\mu_{0}$ are the rotational constant and the permanent dipole moment. $\alpha_{\|}, \alpha_{\perp}, \beta_{\|}$and $\beta_{\perp}$ are respectively the polarizability and the hyperpolarizability components of the molecule. The labels $\|$ and $\perp$ indicate the components parallel and perpendicular to the internuclear axis. For the $C O$ molecule, we have chosen the following numerical values $B=1.9313 \mathrm{~cm}^{-1}$ and $\mu_{0}=0.044, \alpha_{\|}=15.65$, $\alpha_{\perp}=11.73, \beta_{\|}=28.35$ and $\beta_{\perp}=6.64$ in atomic units [46, 47. $J^{2}$ is the angular momentum operator and $\theta$ the angle between the direction of the molecular axis and the polarization vector. A basis of the Hilbert space is given by the spherical harmonics $|j, m\rangle$ with $j \geq 0$ and $-j \leq m \leq j$.

3.2. Zero rotational temperature. In this section, we consider the limit of zero rotational temperature. We recall that the expectation value $\langle\cos \theta\rangle$ is usually taken as a quantitative measure of orientation [28, 29]. Here, we replace this measure by the projection onto a target state $\left|\phi_{f}\right\rangle$. We consider target states recently introduced for the orientation which both maximize the field-free orientation and its duration [32, 31]. To construct this target state, we restrict the Hilbert space to a finite-dimensional one defined by a maximum value of $j$ denoted $j_{\text {opt }}$. For $C O$, we have chosen $j_{\text {opt }}=4$ which leads to a maximum of $\langle\cos \theta\rangle$ of the order of 0.9. In this reduced Hilbert space, the operator $\cos \theta$ has a non-degenerate discrete spectrum. The target state $\left|\phi_{f}\right\rangle$ is then defined as the eigenvector of $\cos \theta$ of highest eigenvalue. The initial state is the state $|0,0\rangle$. We also recall that the projection $m$ of the angular momentum $j$ on the field polarization axis is a conserved quantum number due to cylindrical symmetry.

We now apply the monotonically convergent algorithms I and II. The results of the computations are presented in Figs. 1, 2, 3) and 4. We have used the simplified algorithms by assuming that $E_{k}=\tilde{E}_{k}$. Figures 1, 3 and 4 correspond respectively to the algorithm II for $n=1$, the algorithm II for $n=2$ and the algorithm I for $n=2$. For the algorithm II, we can choose $n=1$ since we have checked that for this value Eqs. (24) and (25) always have a real solution. Note that this latter observation depends on the values of $\lambda$ and $\eta$ considered. Numerical values are taken to be $\lambda=0.05, \eta=1$ for Fig. 10 ( $\lambda$ corresponds to the maximum value of $\lambda(t)), \lambda=6.05 \times 10^{4}, \eta=1$ for Fig. 3 and $\lambda=12 \times 10^{5}$ for Fig. 4. The difference in the values of $\lambda$ is due to the form of the cost which is either quadratic or quartic in the field. We have checked that the value of $\eta$ is not relevant even if the value of $\lambda$ has to be adjusted with respect to the one of $\eta$. The trial fields are displayed in Fig. 1 $\mathrm{a}, 3 \mathrm{a}$ and $4 \mathrm{a}$. The trial field is a gaussian pulse of intensity of the order of $1 \mathrm{TW} / \mathrm{cm}^{2}$. In order to obtain realistic electric fields, the value of $\lambda$ has been chosen so that the energy of the optimal field be lower than two times the energy 

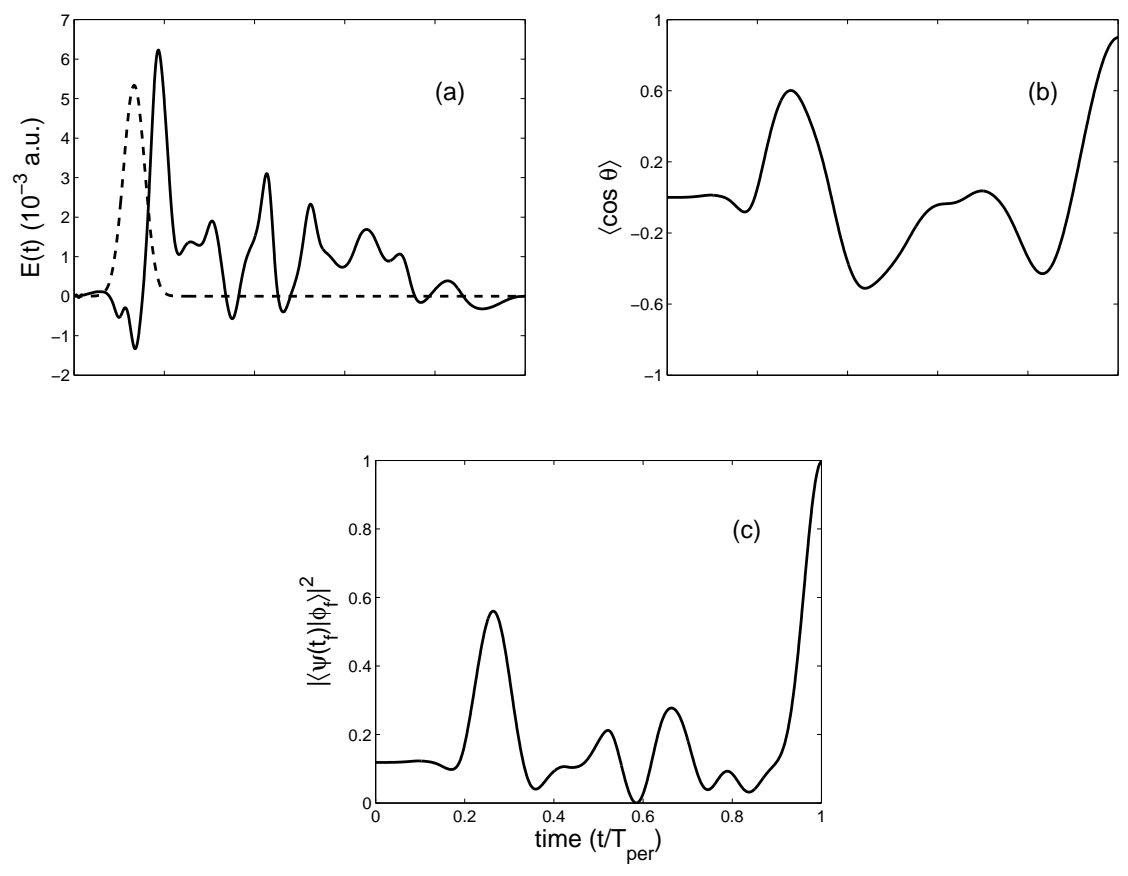

Figure 1. Plot as a function of the adimensional time $t / T_{\text {per }}$ of (a) the optimal field (solid line) and the initial trial field (dashed line), (b) the expectation value $\langle\cos \theta\rangle$ and (c) the projection onto the target state $\left|\phi_{f}\right\rangle$. The abbreviation a.u. corresponds to atomic units.
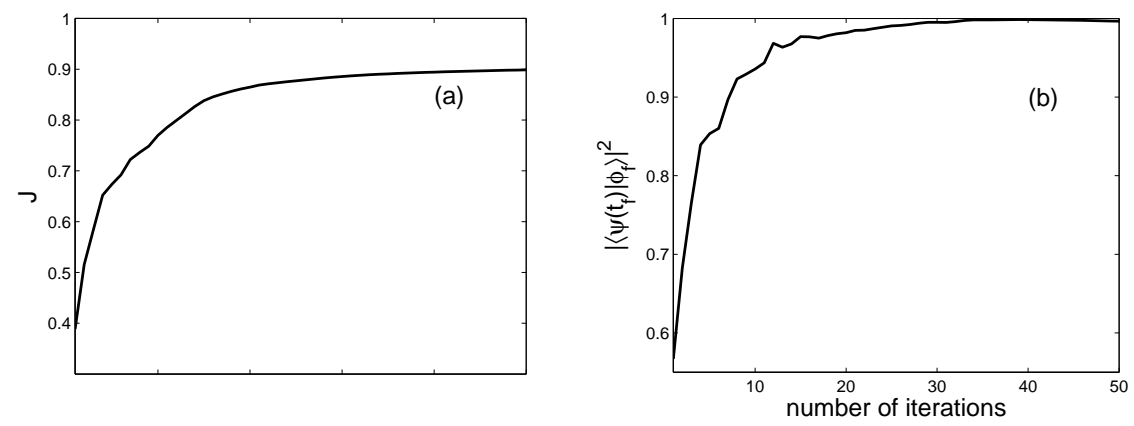

Figure 2. Plot as a function of the number of iterations of (a) the adimensional cost $J$ defined by Eq. (12) for $n=1$ (a cost quadratic in the field) and (b) the projection onto the target state at time $t_{f}$.

of the gaussian pulse. Note also that for $E \simeq 5 \cdot 10^{-3}$ a.u. (which corresponds to the typical amplitude of the optimal field), we have $\mu_{0} \simeq \alpha_{\perp} E$ which shows that the polarizability terms are not negligible in the dynamics. In each case, very good results are obtained with a final projection $\left|\left\langle\psi\left(t_{f}\right) \mid \phi_{f}\right\rangle\right|^{2}$ larger than 0.99 except 

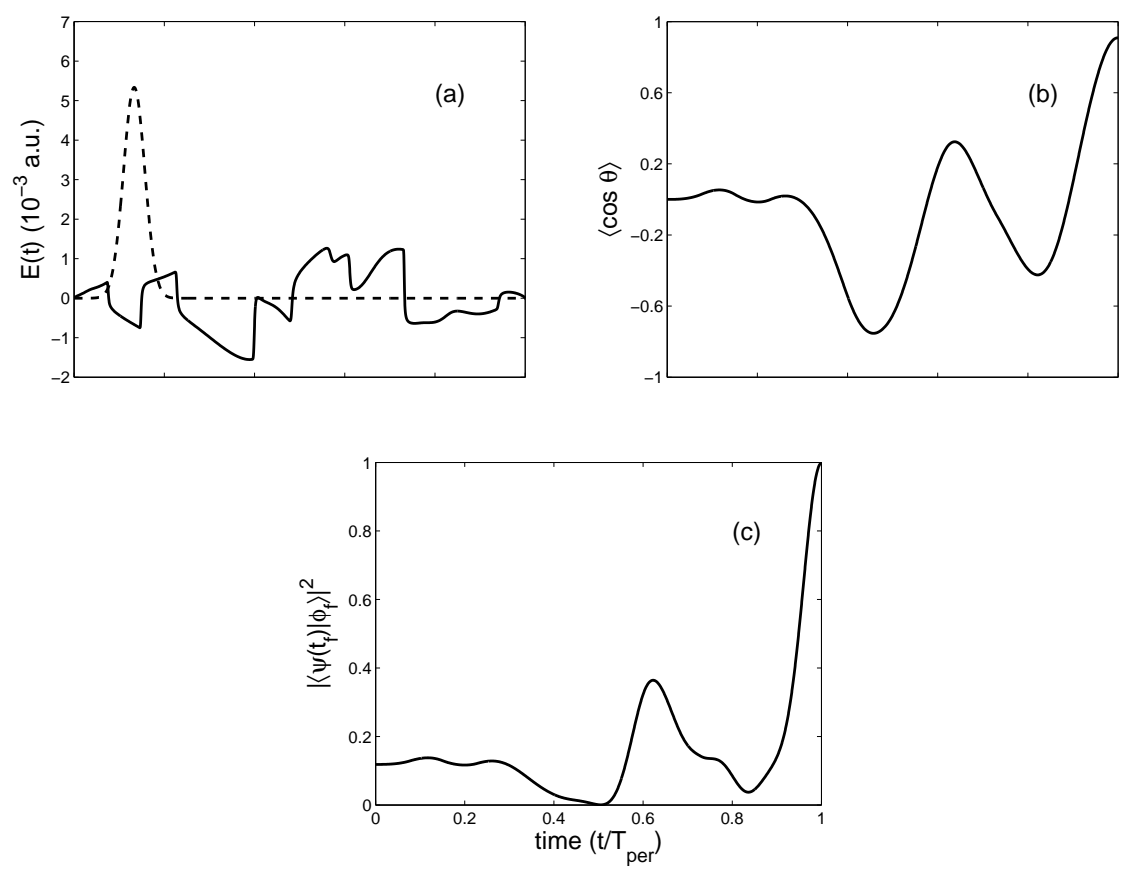

Figure 3. Same as Fig. 1 but for $n=2$, i.e., a cost quartic in the field.
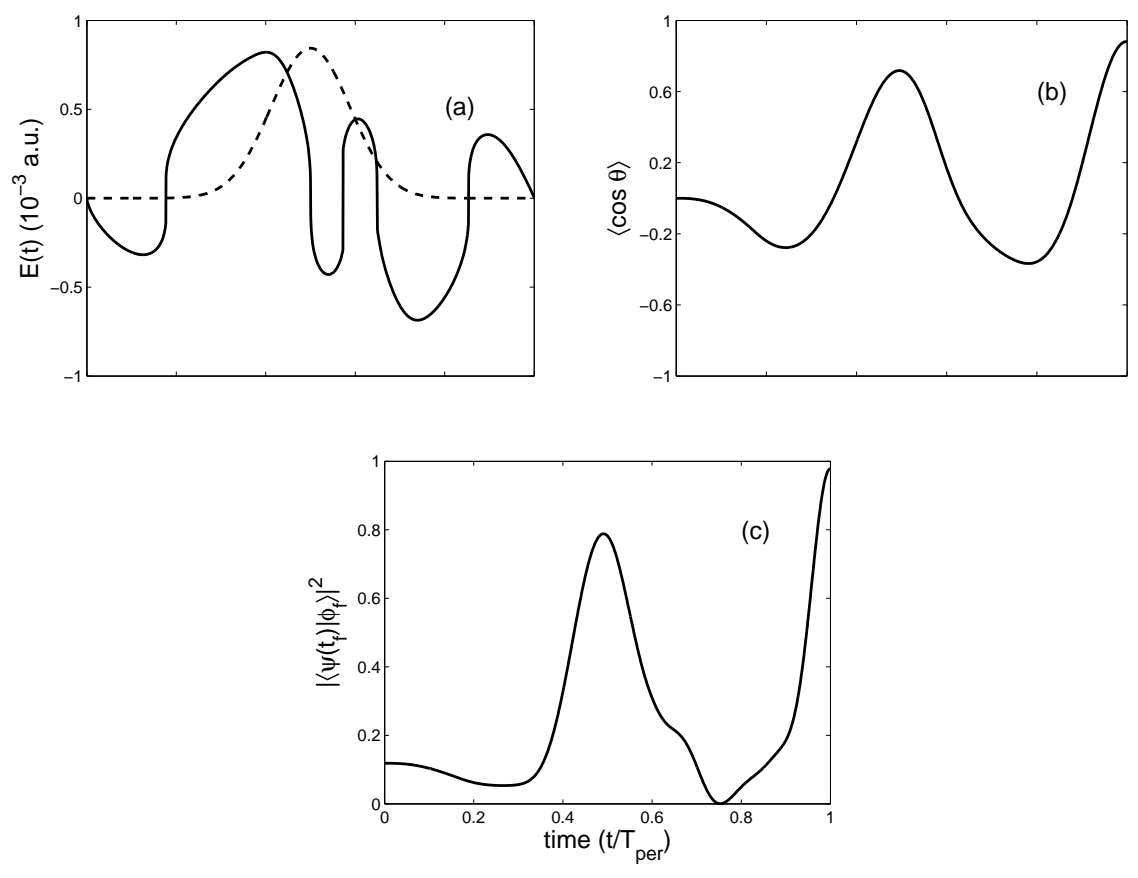

Figure 4. Same as Fig. 1 but for the algorithm I and $n=2$. 
for the algorithm I where $\left|\left\langle\psi\left(t_{f}\right) \mid \phi_{f}\right\rangle\right|^{2}$ is of the order of 0.98. Figure 2 illustrates the convergence properties of the algorithm II which are satisfactory since after 30 iterations, we obtain a projection close to 0.98. A similar behavior has been observed in the other cases. A comparison of Figs. 1a, 3a and 4a shows that the optimal field for $n=2$ has sharper variations than for $n=1$ for both algorithms. We have also observed that these sharper variations can induce numerical instabilities and high frequency oscillations in the optimal field. This point is discussed in Sec. 3.4 where we show how to remove the parasite oscillations with a band-pass filter. In practice, it has been found that small values of the exponent $n$ generally produce smoother optimal fields.

3.3. Non-resonant two-color laser fields. We continue to consider a zero rotational temperature but we assume now that the molecule interacts with a nonresonant two-color laser field [24, 27] of the form

$$
E(t)=E_{1}(t) \cos (\omega t)+E_{2}(t) \cos (2 \omega t) .
$$

After averaging over the rapid oscillations of the field, the Hamiltonian $\hat{H}$ of the system becomes

$$
\begin{aligned}
& \hat{H}=B \hat{J}^{2}-\frac{1}{4}\left[\left(\alpha_{\|}-\alpha_{\perp}\right) \cos ^{2} \theta+\alpha_{\perp}\right]\left(E_{1}(t)^{2}+E_{2}(t)^{2}\right) \\
& -\frac{1}{8}\left[\left(\beta_{\|}-3 \beta_{\perp}\right) \cos ^{3} \theta+3 \beta_{\perp} \cos \theta\right] E_{1}(t)^{2} E_{2}(t) .
\end{aligned}
$$

The interest of this model is due to the absence of linear term in the interaction which enhances the difficulty of the control. Two cases can be considered according to the respective values of $E_{1}$ and $E_{2}$. If $E_{1}=E_{2}$, we can use the standard algorithm presented in Sec. 2.3 whereas for $E_{1} \neq E_{2}$, the algorithm has to be slightly generalized. These two problems are respectively analyzed in Secs. 3.3 .1 and 3.3 .2

3.3.1. The case $E_{1} \neq E_{2}$. We first generalized the algorithm of 2.3 to the case of two control fields. We assume that the cost is quadratic in the field.

We introduce the augmented cost $\bar{J}$ and we determine the critical points with respect to $E_{1}$ and $E_{2}$. We have:

$$
\begin{aligned}
& \bar{J}=\left|\left\langle\phi_{f} \mid \psi\left(t_{f}\right)\right\rangle\right|^{2}-\int_{0}^{t_{f}} \lambda\left[E_{1}(t)^{2}+E_{2}(t)^{2}\right] d t \\
& -2 \Im\left[\left\langle\psi\left(t_{f}\right) \mid \phi_{f}\right\rangle \int_{0}^{t_{f}}\left\langle\chi(t)\left|\left(i \frac{\partial}{\partial t}-\hat{H}\right)\right| \psi(t)\right\rangle d t\right],
\end{aligned}
$$

and we compute the variational derivatives $\delta \bar{J} / \delta E_{1}$ and $\delta \bar{J} / \delta E_{2}$ which are equal to zero for a critical point. We then obtained the following system of equations

$$
\begin{aligned}
& \lambda E_{1}+2 \tilde{\alpha} E_{1}+2 \tilde{\beta} E_{1} E_{2}=0 \\
& \lambda E_{2}+2 \tilde{\alpha} E_{2}+\tilde{\beta} E_{1}^{2}=0
\end{aligned}
$$

which are satisfied by the optimal fields $E_{1}$ and $E_{2}$. We have used in Eqs. (30) the notations

$$
\begin{aligned}
& \tilde{\alpha}=2 \Im\left[\langle\psi(t) \mid \chi(t)\rangle\left\langle\chi(t)\left|\frac{1}{4}\left(\left(\alpha_{\|}-\alpha_{\perp}\right) \cos ^{2} \theta+\alpha_{\perp}\right)\right| \psi(t)\right\rangle\right] \\
& \tilde{\beta}=2 \Im\left[\langle\psi(t) \mid \chi(t)\rangle\left\langle\chi(t)\left|\frac{1}{8}\left[\left(\beta_{\|}-3 \beta_{\perp}\right) \cos ^{3} \theta+3 \beta_{\perp} \cos \theta\right]\right| \psi(t)\right\rangle\right] .
\end{aligned}
$$

We solve the optimal equations by a monotonically convergent algorithm. The proof of monotonicity follows closely the lines of proof in Sec. 2.3. We use the 

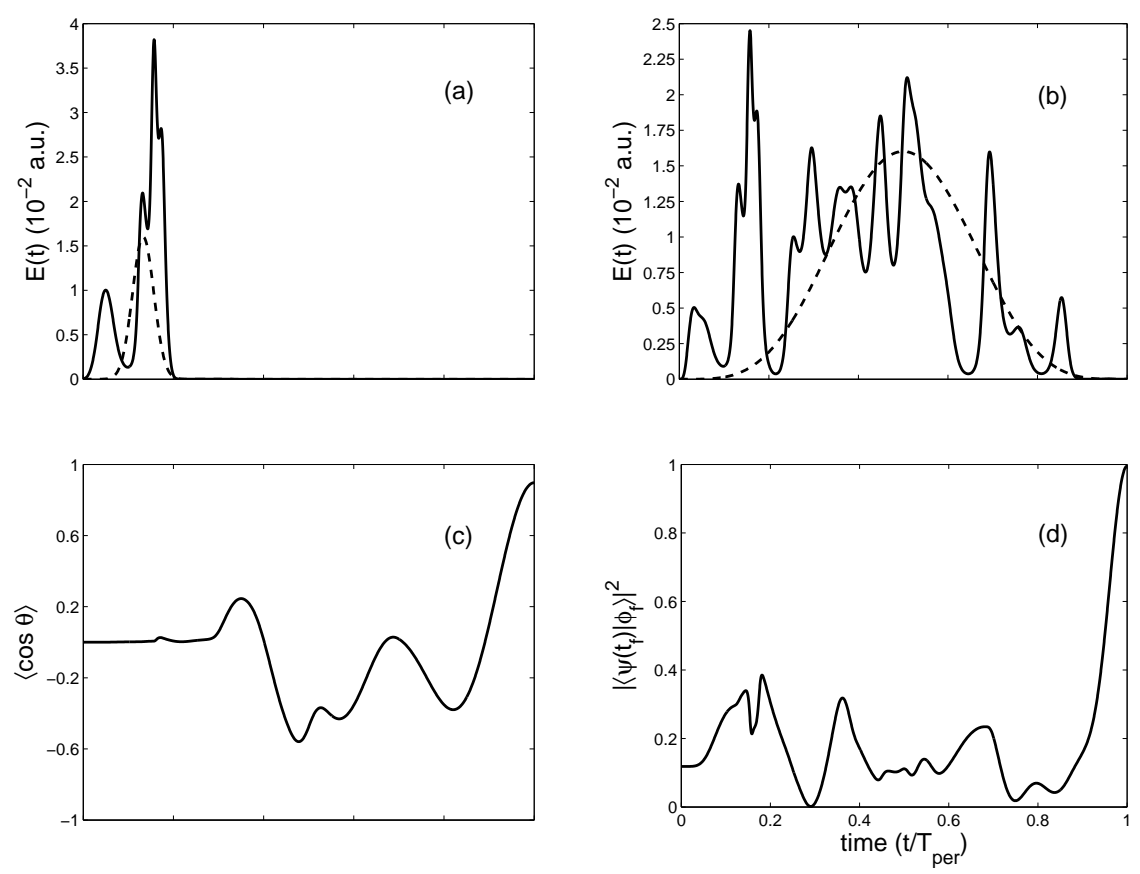

Figure 5. Same as Fig. 1 but for two-color laser fields with $E_{1} \neq E_{2}$. The panels (a) and (b) correspond respectively to the fields $E_{1}$ and $E_{2}$.

same notations, with for instance $E_{1, k}$ the field $E_{1}$ at iteration $k$. To simplify the computations, we take equal the fields $E_{k}$ and $\tilde{E}_{k}$ for the forward and the backward propagations. We compute $\Delta J=J_{k+1}-J_{k}$. We obtain the following expressions for the polynomials $\mathcal{P}_{1}$ and $\mathcal{P}_{2}$ :

$$
\begin{aligned}
& \mathcal{P}_{1}=\left(E_{1, k+1}-E_{1, k}\right)\left[\left(E_{1, k+1}+E_{1, k}\right) \alpha_{k, k+1}+\right. \\
& \left.\left(E_{1, k+1}+E_{1, k}\right) E_{2, k+1} \beta_{k, k+1}-\lambda\left(E_{1, k+1}+E_{1, k}\right)\right]
\end{aligned}
$$

and

$$
\begin{aligned}
& \mathcal{P}_{2}=\left(E_{2, k+1}-E_{2, k}\right)\left[\left(E_{2, k+1}+E_{2, k}\right) \alpha_{k, k+1}+\right. \\
& \left.E_{1, k}^{2} \beta_{k, k+1}-\lambda\left(E_{2, k+1}+E_{2, k}\right)\right]
\end{aligned}
$$

where

$$
\begin{aligned}
\alpha_{k}, k+1 & =\Im\left[\left\langle\psi_{k+1}(t) \mid \chi_{k}(t)\right\rangle\left\langle\chi_{k}(t)\left|\frac{1}{4}\left(\left(\alpha_{\|}-\alpha_{\perp}\right)+\alpha_{\perp}\right) \cos ^{2} \theta\right| \psi_{k+1}(t)\right\rangle\right] \\
\left(3 \beta_{k, k+1}\right. & =\Im\left[\left\langle\psi_{k+1}(t) \mid \chi_{k}(t)\right\rangle\left\langle\chi_{k}(t)\left|\frac{1}{8}\left[\left(\beta_{\|}-3 \beta_{\perp}\right) \cos ^{3} \theta+3 \beta_{\perp} \cos \theta\right]\right| \psi_{k+1}(t)\right\rangle\right] .
\end{aligned}
$$

$\mathcal{P}_{1}$ and $\mathcal{P}_{2}$ are respectively viewed as polynomials in $E_{1, k+1}$ and $E_{2, k+1}$. We first use $\mathcal{P}_{2}$ to determine the field $E_{2, k+1}$ by the algorithm I or II and then using this solution, we compute $E_{1, k+1}$ from $\mathcal{P}_{1}$. We also check that if the algorithm converges then the solutions given by the algorithm correspond to the extremal solutions defined by Eqs. (30). This can be done by replacing $E_{1, k+1}$ and $E_{1, k}$ by $E_{1}$ and $E_{2, k+1}$ and $E_{2, k}$ by $E_{2}$. The optimal fields are then zeros of the derivatives of $\mathcal{P}_{1}$ and $\mathcal{P}_{2}$ with respect to $E_{1, k+1}$ and $E_{2, k+1}$. Figure 5 displays the results we have obtained with the algorithm II for $n=2$ and $\lambda=1$. We have chosen two different trial fields in 

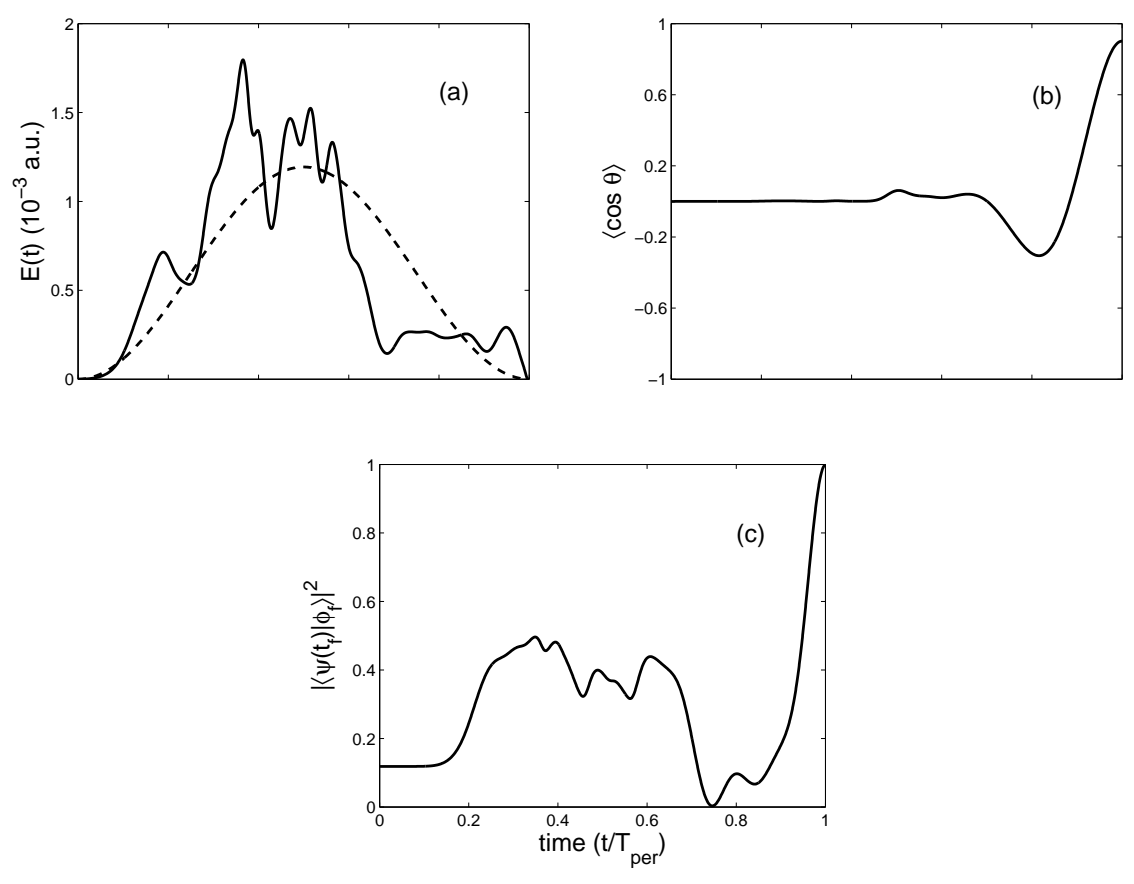

Figure 6. Same as Fig. 1 but for the averaging case with $E_{1}=E_{2}$.

order to generate two different optimal fields $E_{1}$ and $E_{2}$. With the same trial field for the fields $E_{1}$ and $E_{2}$, the algorithm leads to two solutions which are very close to each other. Larger values of electric fields have been used due to the absence of linear interaction term in the Hamiltonian.

A remarkable characteristic of the optimal fields is the fact that $E_{1}$ vanishes for $t>0.2 \times T_{\text {per }}$. This means that the dissymmetry producing the orientation (dissymmetry due to the term in $E_{1}^{2} E_{2}$ in the Hamiltonian) only acts during this duration. This provides a non-intuitive and new method to produce orientation using a long laser field $E_{2}$ and a short laser field $E_{1}$.

3.3.2. The case $E_{1}=E_{2}$. We use the monotonic algorithm II proposed in Sec. 2. Figure 6 illustrates the different results. They have been obtained for $n=2$ and $\lambda=5$. The trial field is a gaussian pulse whose duration corresponds to the rotational period. From the equations of the algorithm, it is straightforward to see that the algorithm cannot generate an optimal field different from zero at time $t$ if the trial field is zero at that time. This is simply due to the absence of linear interaction term in the Hamiltonian. The algorithm only modifies the envelope of the trial field whose choice is therefore crucial.

3.4. Analysis of the Fourier spectrum. We analyze in this section the Fourier transforms of the optimal solutions. Our goal is to show that optimal solutions determined in Sec. 2.1] can be well-approximated by solutions that could be implemented experimentally. We consider experiments coupled with genetic algorithms optimizing the phase and the amplitude of the Fourier transform of a finite number of frequency components. The discretization is done over a frequency interval 

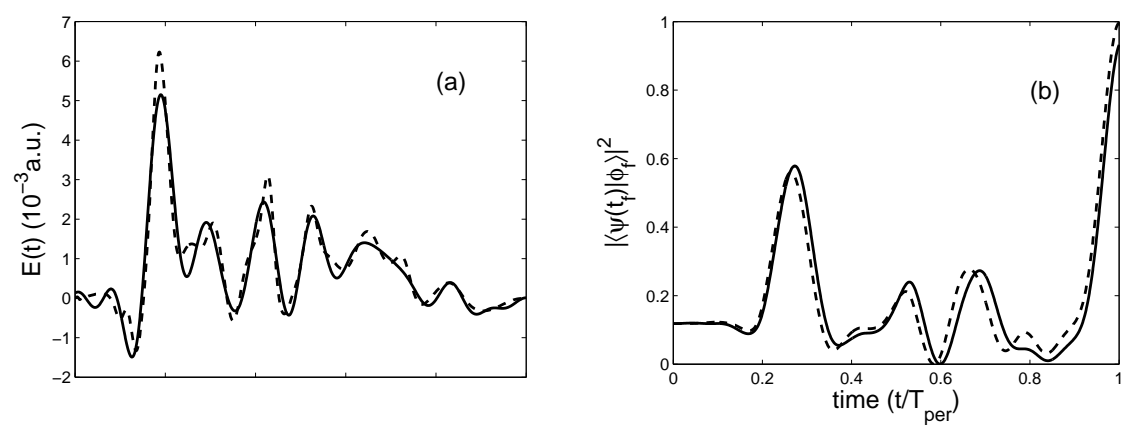

Figure 7. Plot as a function of time $t / T_{\text {per }}$ of (a) the optimal (dashed line) and of the approximate fields (solid line) and (b) the projection onto the target state $\left|\phi_{f}\right\rangle$.
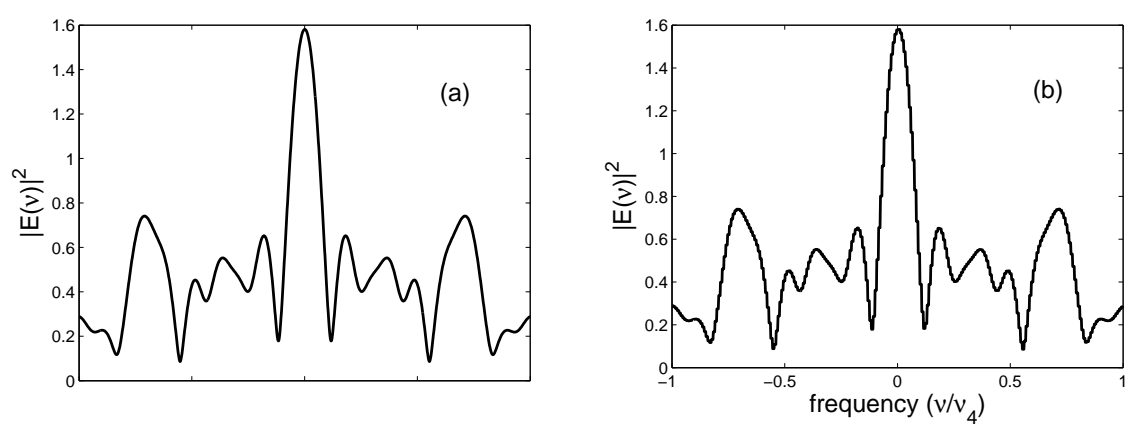

Figure 8. Plot as a function of the frequency $\nu$ of the module square of the Fourier transform of the optimal field (a) and of its piecewise constant approximation (b). The Fourier transform has been discretized over the interval $\left[-\nu_{4}, \nu_{4}\right]$.

chosen with respect to the quantum transition frequencies involved in the control (see below). Note that we do not take into account, in this paper, technology constraints for the choice of this frequency interval. Standard pulse shapers usually work with optical frequencies of the order of $800 \mathrm{~nm}$. With such a technology, only non-resonant laser fields of Sec. 3.3 could be experimentally implemented.

Following 40, 41, we assume that the solution obtained by genetic algorithms is a piecewise constant function in frequency both in amplitude and in phase. We have chosen 640 frequencies or less to discretize the optimal field. By an inverse Fourier transform, we then determine a new time-dependent electric field. Figure 7 presents the results obtained with the optimal pulse of Fig. 1. Using only 128 frequencies, we show that the final projection obtained by the optimal pulse and its approximation are very close to each other. For 256 frequencies, the difference is negligible and cannot be distinguished at the resolution of the plots. Figures 8 and 9 give informations on the Fourier transform of the optimal pulse. One introduces the rotational frequencies $\nu_{j+1}$ given by:

$$
\nu_{j+1}=E_{j+1}-E_{j}=2 B(j+1)
$$




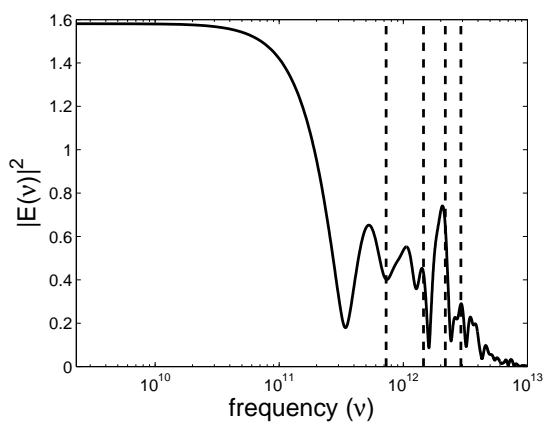

Figure 9. Same as Fig. 8 a but as a function of the frequency $\nu$. Vertical lines indicate the positions of the frequencies $\nu_{1}, \nu_{2}, \nu_{3}$ and $\nu_{4}$.

where $E_{j}$ is the energy of the state $|j, 0\rangle$. The target state being associated to $j_{\text {opt }}=4$, only five rotational states from $j=0$ to $j=4$ have to be populated by the control field. It is thus natural to discretize the Fourier transform over the interval $\left[-\nu_{4}, \nu_{4}\right]$. Higher frequencies do not contribute to reach the target state. A similar behavior has been observed for the other optimal solutions. Another example is given by Fig. 10. The corresponding optimal solution obtained by the algorithm I presents rapid unwanted oscillations. To obtain a smooth solution displayed in Fig. $10 \mathrm{~b}$, we filter this optimal pulse in the frequency domain. The bandwidth of the filter is chosen to cut off frequencies higher than $\nu_{4}$ which produce rapid oscillations. As in the first case, we also observe that the discretization does not significantly modify the final result.

3.5. Finite rotational temperature. We investigate the temperature effects on the optimal solutions. The system is described by a density matrix $\rho$ whose dynamics is governed by the von Neumann equation. The initial density operator $\rho(0)$ is the equilibrium density operator at temperature $T$ which can be written

$$
\rho(0)=\frac{1}{Z} \sum_{j=0}^{+\infty} \sum_{m=-j}^{j} e^{-B j(j+1) /\left(k_{B} T\right)}|j, m\rangle\langle j, m|
$$

where $k_{B}$ is the Boltzmann constant and $Z$ the partition function. The objective of the control is to maximize the projection of $\rho\left(t_{f}\right)$ onto a target state $\rho_{\text {opt }}$. We consider here the target state introduced in Ref. [33] which is unitarily equivalent to the initial mixed state $\rho\left(t_{i}\right)$ and optimizes both the orientation and its duration. We refer the reader to Ref. 33 for the complete construction of $\rho_{\text {opt }}$ and for proofs of its attainability by unitary controls. Note that the definition of $\rho_{\text {opt }}$ depends on the polarization used. We consider here the optimum for a linear polarization. $\rho_{\text {opt }}$ can be defined as follows. The first step consists in reducing the dimension of the Hilbert space to a finite one $\mathcal{H}^{\left(j_{o p t}\right)}$ where $j_{\text {opt }}$ is the highest $j$ for which the corresponding rotational levels are significantly populated. The dimension of this space depends on the temperature and on the intensity of the field used. For $C O$ and $T=1,5$ and $10 \mathrm{~K}$, we have chosen $j_{\text {opt }}=4$. We denote by $\mathcal{H}_{m}^{\left(j_{o p t}\right)}$ the subspace of $\mathcal{H}^{\left(j_{\text {opt }}\right)}$ associated to a given value of $m$. The target state $\rho_{\text {opt }}^{\left(j_{\text {opt }}\right)}$ of the 

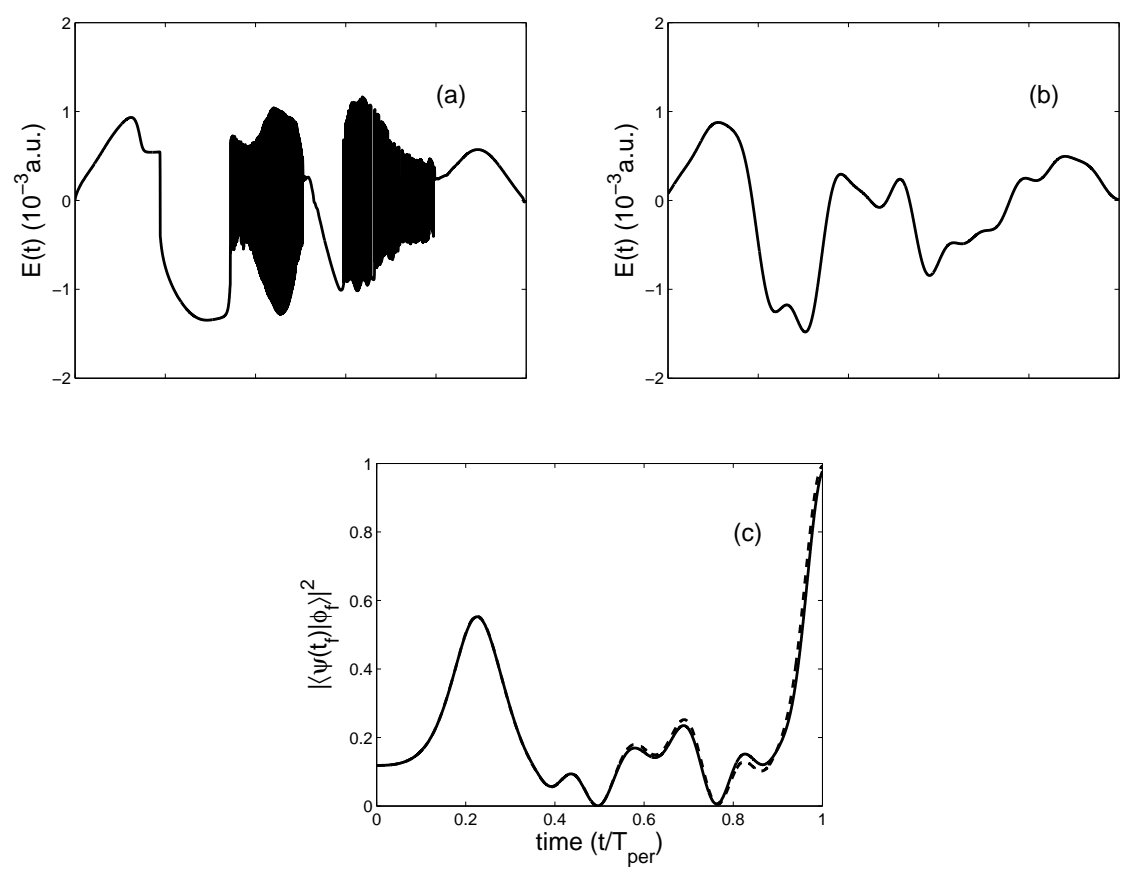

Figure 10. Plot as a function of time $t / T_{p e r}$ of (a) the optimal (solid line) and (b) the approximate fields (dashed line) and (c) the projection onto the target state $\left|\phi_{f}\right\rangle$.

control, which therefore depends on the choice of $j_{\text {opt }}$, is given by

$$
\rho_{\text {opt }}^{\left(j_{\text {opt }}\right)}=\sum_{m=-j_{\text {opt }}}^{m=j_{\text {opt }}} \sum_{k=1}^{j_{o p t}-|m|+1} \omega_{k}^{(m)}\left|\chi_{k}^{(m)}\right\rangle\left\langle\chi_{k}^{(m)}\right|,
$$

where the $\omega_{k}^{(m)}$ 's are the eigenvalues of $\rho\left(t_{i}\right)$ restricted to $\mathcal{H}_{m}^{\left(j_{o p t}\right)}$ and ordered. The vectors $\left|\chi_{k}^{(m)}\right\rangle$ are the eigenvectors of the restriction of the operator $\cos \theta$ to $\mathcal{H}_{m}^{\left(j_{\text {opt }}\right)}$. The vectors $\left|\chi_{k}^{(m)}\right\rangle$ are also ordered according to the values of the corresponding eigenvalues.

We have used the algorithm II with $n=2$ to determine the optimal solutions. We denote by $\chi(t)$ the adjoint density matrix state. In superoperator notations, the structure of the algorithm is very similar to the one for pure states. The cost functional $J$ is given by

$$
J=\left|\left\langle\left\langle\rho_{\text {opt }} \mid \rho\left(t_{f}\right)\right\rangle\right\rangle\right|^{2}-\int_{0}^{t_{f}} \lambda E(t)^{4} d t
$$

and the augmented cost functional $\bar{J}$ reads

$$
\begin{aligned}
\bar{J}=\quad & \left|\left\langle\left\langle\rho_{\text {opt }} \mid \rho\left(t_{f}\right)\right\rangle\right\rangle\right|^{2}-2 \Im\left[\left\langle\left\langle\rho\left(t_{f}\right) \mid \rho_{\text {opt }}\right\rangle\right\rangle \int_{0}^{t_{f}}\left\langle\left\langle\chi(t)\left|\left(i \frac{\partial}{\partial t}-\hat{H}\right)\right| \rho(t)\right\rangle\right\rangle d t\right] \\
& -\int_{0}^{t_{f}} \lambda E(t)^{4} d t
\end{aligned}
$$



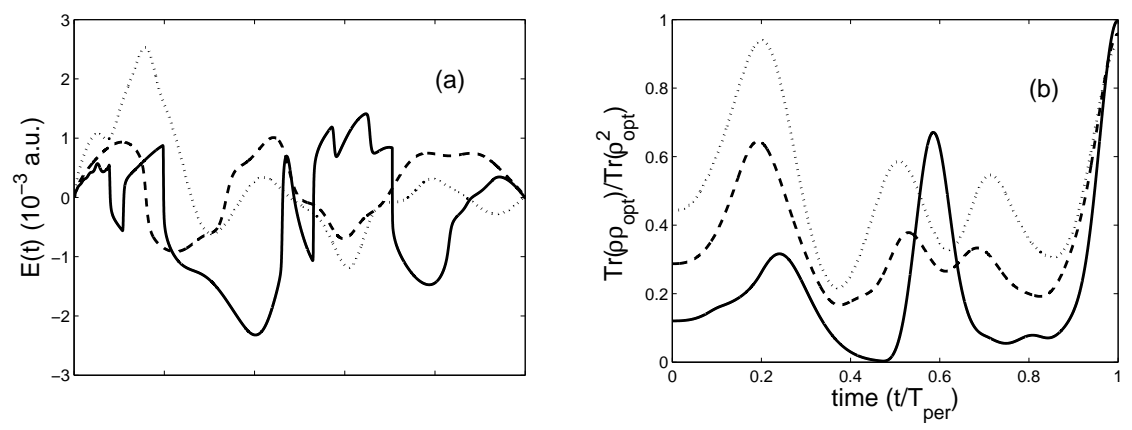

Figure 11. Plot as a function of the adimensional time $t / T_{\text {per }}$ of (a) the optimal electric field and (b) the projection onto the target state $\rho_{\text {opt }}$. Solid, dashed and dot-dashed lines correspond respectively to $T=1 \mathrm{~K}, T=5 \mathrm{~K}$ and $T=10 \mathrm{~K}$.

where $\langle\langle\chi \mid \rho\rangle\rangle=\operatorname{Tr}\left[\chi^{\dagger} \rho\right]$ and $\langle\langle\chi|M| \rho\rangle\rangle=\operatorname{Tr}\left[\chi^{\dagger}[M, \rho]\right]$ for a given observable $M$. $\rho(t)$ and $\chi(t)$, which satisfy the von Neumann equation, are propagated forward and backwards with initial condition $\rho(0)=\rho_{0}$ and final condition $\chi\left(t_{f}\right)=\rho_{\text {opt }}$. Numerical parameters are respectively taken to be $\lambda=6.10^{4}, \lambda=65.10^{2}$ and $\lambda=90$ for $T=1 \mathrm{~K}, T=5 \mathrm{~K}$ and $T=10 \mathrm{~K}$. $\eta$ is equal to 1 in all the cases. The values of $\lambda$ are chosen so that the total energy of the field stays approximatively constant when the temperature is increased. The trial field is the same for the three cases considered.

Figure 11 presents the results obtained for three different temperatures. We observe that the structures of the optimal fields and of the projection onto the target state as a function of time are very different. As expected, we note a decrease of the final projection with increasing temperature. This computation allows us to show the robustness with respect to temperature of the optimal fields. For $T=10 \mathrm{~K}$, we still obtain an efficient field since the final projection is of the order of 0.9 . We also point out sharp variations of the optimal fields due to the use of a cost that is quartic in the control field. These variations do no affect the temporal evolution of the projection.

\section{Summary}

We have presented a new family of monotonically convergent algorithms for the computation of the optimal control of a quantum system interacting non-linearly with the laser field. One key for the convergence of these algorithms is to consider costs which are not quadratic in the field. In comparison with algorithms of Ref. [12, this allows to consider only one wave function and one adjoint state per iteration of the algorithm whatever the nonlinearity used. This is thus less demanding from a numerical point of view especially when the degree of the nonlinearity is important. As a prospect, an open question in this field is the applicability of the present method to more complicated systems involving, for instance, non-markovian dynamics or a time-dependent target state [48]. Special attention has to be paid to the convergence properties and to the stability of the method, especially when the cost is quartic in the control field. 


\section{ACKNOWLEDGMENTS}

We thank E. Hertz for many helpful discussions. We acknowledge support from the Agence Nationale de la recherche (ANR CoMoc, C-QUID). G.T. acknowledges support from INRIA Rocquencourt (MicMac project) and a PICS CNRS-NSF program on quantum control.

\section{REFERENCES}

1. W. Warren, H. Rabitz, and M. Dahleb, Science 259, 1581 (1993).

2. H. Rabitz, R. de Vivie-Riedle, M. Motzkus, and K. Kompa, Science 288, 824 (2000).

3. M. A. Nielsen and I. L. Chuang, Quantum Computation and Quantum Information (Cambridge University Press, Cambridge, 2000).

4. W. Zhu and H. Rabitz, J. Chem. Phys. 109, 385 (1998).

5. Y. Maday and G. Turinici, J. Chem. Phys. 118, 8191 (2003).

6. S. Schirmer, M. Girardeau, and J. Leahy, Phys. Rev. A 61, 012101 (2000).

7. Y. Ohtsuki, W. Zhu, and H. Rabitz, J. Chem. Phys. 110, 9825 (1999).

8. Y. Ohtsuki, G. Turinici, and H. Rabitz, J. Chem. Phys. 120, 5509 (2004).

9. B. Bonnard and D. Sugny, Optimal control with applications in space and quantum dynamics (AIMS Applied Maths, submitted, 2008).

10. D. Sugny, C. Kontz, and H. Jauslin, Phys. Rev. A 76, 023419 (2007a).

11. J. Werschnik and E. K. U. Gross, J. Phys. B 40, R175 (2007).

12. Y. Ohtsuki and K. Nakagami, Phys. Rev. A 77, 033414 (2008).

13. H. Jirari and W. Potz, Phys. Rev. A 72, 013409 (2005).

14. M. Wenin and W. Potz, Phys. Rev. A 74, 022319 (2006).

15. A. E. Bryson and Y. Ho, Applied optimal control (Hemisphere Publishing Corporation, Washington, 1975).

16. R. Kosloff, S. Rice, P. Gaspard, S. Tersigni, and D. Tannor, Chem. Phys. 139, 201 (1989).

17. W. Zhu, J. Botina, and H. Rabitz, J. Chem. Phys. 108, 1953 (1998).

18. W. Zhu and H. Rabitz, J. Chem. Phys. 110, 7142 (1999).

19. M. Ndong, L. Bomble, D. Sugny, Y. Justum, and M. Desouter-Lecomte, Phys. Rev. A 76, $043424(2007)$.

20. D. Sugny, C. Kontz, M. Ndong, Y. Justum, G. Dives, and M. Desouter-Lecomte, Phys. Rev. A 74, 043419 (2006a).

21. D. Sugny, M. Ndong, D. Lauvergnat, Y. Justum, and M. Desouter-Lecomte, J. Photochem. Photobiol. A : Chemistry 190, 359 (2007b).

22. J. Salomon, C. M. Dion, and G. Turinici, J. Chem. Phys. 123, 144310 (2005).

23. Y. Ohtsuki, Y. Teranishi, P. Saalfrank, G. Turinici, and H. Rabitz, Phys. Rev. A 75, 033407 (2007).

24. B. Friedrich and D. Herschbach, Phys. Rev. Lett. 74, 4623 (1995).

25. B. J. Sussman, M. Y. Ivanov, and A. Stolow, Phys. Rev. A 71, R051401 (2005).

26. J. G. Underwood, M. Spanner, M. Y. Ivanov, J. Mottershead, B. J. Sussman, and A. Stolow, Phys. Rev. Lett. 90, 223001 (2003).

27. R. Tehini and D. Sugny, Phys. Rev. A 77, 023407 (2008).

28. H. Stapelfeldt and T. Seideman, Rev. Mod. Phys. 75, 543 (2003).

29. T. Seideman and E. Hamilton, Adv. At. Mol. Opt. Phys. 52, 289 (2006).

30. G. Turinici, in Control of coupled partial differential equations (Birkhäuser, Basel, 2007), vol. 155 of Internat. Ser. Numer. Math., pp. 293-309.

31. D. Sugny, A. Keller, O. Atabek, D. Daems, C. M. Dion, S. Guérin, and H. R. Jauslin, Phys. Rev. A 69, 033402 (2004a).

32. D. Sugny, A. Keller, O. Atabek, D. Daems, C. M. Dion, S. Guérin, and H. R. Jauslin, Phys. Rev. A 71, 063402 (2005a).

33. D. Sugny, A. Keller, O. Atabek, D. Daems, C. M. Dion, S. Guérin, and H. R. Jauslin, Phys. Rev. A 72, 032704 (2005b).

34. D. Sugny, C. Kontz, and H. Jauslin, Phys. Rev. A 74, 053411 (2006b).

35. R. S. Judson and H. Rabitz, Phys. Rev. Lett. 68, 1500 (1992).

36. A. Assion, T. Baumer, M. Bergt, T. Brixner, B. Kiefer, V. Seyfried, M. Strehle, and G. Gerber, Science 282, 919 (1998). 
MONOTONICALLY CONVERGENT OPTIMAL CONTROL THEORY OF QUANTUM SYSTEMS UNDER A NONLINEAR INTERA

37. R. J. Levis, G. M. Menkir, and H. Rabitz, Science 292, 709 (2001).

38. C. Daniel, J. Full, L. Gonzàlez, C. Lupulescu, J. Manz, A. Merli, S. Vadja, and L. Woste, Science 299, 536 (2003).

39. O. M. Shir, V. Beltrani, T. Back, H. Rabitz, and M. Vrakking, J. Phys. B 41, 074021 (2008).

40. E. Hertz, A. Rouzée, S. Guérin, B. Lavorel, and O. Faucher, Phys. Rev. A 75, 031403 (2007).

41. A. Rouzée, E. Hertz, B. Lavorel, and O. Faucher, J. Phys. B 41, 074002 (2008).

42. K. Sundermann and R. de Vivie-Riedle, J. Chem. Phys. 110, 1896 (1999).

43. A. Pelzer, S. Ramakrishna, and T. Seideman, J. Chem. Phys. 126, 034503 (2007).

44. D. Sugny, A. Keller, O. Atabek, D. Daems, S. Guérin, and H. R. Jauslin, Phys. Rev. A 69, 043407 (2004b).

45. T. Kanai and H. Sakai, J. Chem. Phys. 115, 5492 (2001).

46. H. Sekino and R. J. Bartlett, J. Chem. Phys. 98, 3022 (1993).

47. G. Maroulis, J. Phys. Chem. 100, 13466 (1996).

48. I. Serban, J. Werschnik, and E. K. U. Gross, Phys. Rev. A 71, 053810 (2005).

1 Institut Carnot de Bourgogne, UMR 5209 CNRS-Université de Bourgogne, BP 47870, 21078 DiJON, France

2 Ceremade, Université Paris Dauphine, Place du Maréchal De Lattre De TasSigny, 75775 Paris Cedex 16, France 